\title{
Chitosan/hyaluronic acid membrane funcionalized with gold nanorods by layer-by-layer technique characterized by photothermal activity
}

\section{Kleber E. V. Santos*, Marisa M. Beppu}

\begin{abstract}
Hyperthermia therapy, i.e., the destruction of tissue activity by heating can be used in cancer treatment. The use of gold nanotubes can produce this hyperthermia specific to the tissue reached by tumor. Using LbL technique and aimimg drug delivery, thin films were produced by chitosan/hyaluronic acid LBL and functionalized with gold nanoparticles. The films were characterized by UV-Vis, AFM, SEM-EDS.
\end{abstract}

Key words: Gold, nanoparticles, natural polimers.

\section{Introduction}

The production of thin films by layer-by-layer (LbL) technique presents significant versatility due to the variability both in materials and parameters used in the assembly process. This technique allows films to be formed by deposition of layers of opposite charges on a substrate, so that the molecular organization depends on parameters such as: materials, $\mathrm{pH}$ conditions and ionic forces of the polyelectrolyte solutions. For composite films of the polyelectrolytes hyaluronic acid $(\mathrm{HA})$ and chitosan $(\mathrm{CHI})$, both natural polymers, the electrostatic interaction can be highlighted as the main driving force for multilayer construction. In this context, this project aimed to investigate the photothermal relationship for $\mathrm{HA} / \mathrm{CHI}$ films by $\mathrm{LbL}$ functionalized with gold nanorod (AuNRs).

\section{Results and Discussion}

In order to incorporate gold into the polymer matrix, two possibilities were studied: (i) incorporation of already stabilized nanoparticles by immersing PEM in gold nanoparticle suspension; (ii) production in situ of gold nanoparticles in polymer matrix. Gold nanoparticles (AuNP) were synthetized instead of AuNRs due to its easy of make procedure and stability.

(i) Films of $(\mathrm{HA} / \mathrm{CHI} 3) 3$ and $(\mathrm{HA} / \mathrm{CHI} 3) 3.5$ (Chart 1) were produced which were then immersed in suspension of gold nanoparticles for enough time to changes not to be noticed anymore. Characterization of this films by scanning electron microscopy with an energy dispersive Xray microanalysis system (SEM-EDS) demonstrate that the best choice was $(\mathrm{HA} / \mathrm{CH} / 3) 3$ since dispersion of gold was homogeneous and the other option shows no gold in the surface at all. This aspect could be understood by comparing superficial charges of the polymers and gold nanoparticles. $\mathrm{CHI}$, on top, presents positive charge that interact attractively with gold nanoparticles that presents a negative citrate shell. HA presents a negative charge and presents no attraction by gold nanoparticles.

(ii) In order to observe the mechanisms of interaction of gold with the polymer matrix dyeing free functional groups technique was used: $\mathrm{CHI}$ (amino groups) using Bengal Rose anionic stain (BR) and free HA groups (Carboxyl groups) with cationic dye Alcian Blue (AB). Two parameters were studied: number of bilayers ( 3 and 30 ), immersion time in the gold solution ( 30 and $3600 \mathrm{~s}$ ).
From Image 1, it is clear that 30 bilayers show a more characteristic spectrum of $A B$ absorption and the time dependency of gold incorporation. The same can be stated for BR dyeing. In addition, atomic force microscopy (AFM), Image 2, clarify the particles on the surface of the films.

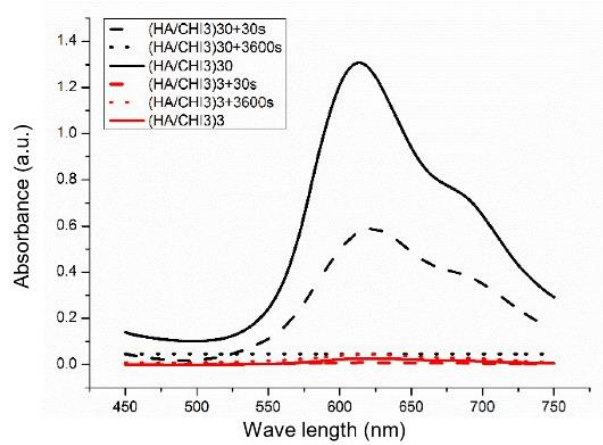

Image 1. UV-VIS spectrum of PEMs dyed with $A B$.

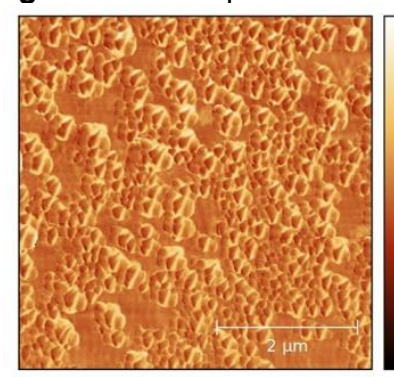

(HA/CHI3) $30+3600$

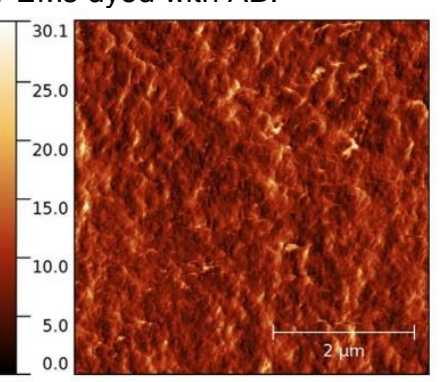

$(\mathrm{HA} / \mathrm{CHI} 3) 30$
Image 2. AFM phase image of PEMs.

Chart 1. Nomenclatures.
\begin{tabular}{|l|l}
\hline$(\mathrm{HA} / \mathrm{CHI}<\mathrm{pH}>) \mathrm{N}$ & $\langle\mathrm{pH}>: \mathrm{pH}$ of polymer solution
\end{tabular}

\begin{tabular}{l|l|}
\cline { 2 - 2 }$(\mathrm{HA} / \mathrm{CHI}<\mathrm{pH}>) \mathrm{N}+\mathrm{T}$ & $\mathrm{N} \mathrm{N}: \mathrm{pH}$ of polymer solution \\
\hline
\end{tabular}

Conclusions

In situ synthesis of gold on a LBL film with $\mathrm{CHI}$ on top demonstrate best results. This technique can be used in future studies to functionalization of LbL films with AuNRs.

\section{Acknowledgement}

To Lequip laboratory co-workers, for the help and coffee time. To CNPq, for the financial support.

${ }^{1}$ Singh M, Harris-Birtill DC, Zhou Y, Gallina ME, Cass AE, Hanna GB, Elson DS. Application of gold nanorods for photothermal therapy in Ex Vivo human oesophagogastric adenocarcinoma. Journal of biomedical nanotechnology. 2016 Mar 1;12(3):481-90. DOI: 10.19146/pibic-2017-79034 